\title{
Bar-Induced Shocks and the Nature of Regions of Violent Star Formation
}

\author{
A. D. Chernin \\ Sternberg Astronomical Institute, Moscow University, Moscow, 119899, \\ Russia
}

\begin{abstract}
Gas dynamics induced by bars involves large-scale shocks. The shock fronts may be curved, especially near the bar edges. Evolution of the curved shocks can lead to effective compression of the interstellar gas which produces favorable conditions for violent star formation in the vicinity of bars.
\end{abstract}

\section{Introduction}

Spectacular star forming regions have been observed near bars in a number of galaxies. The ends of many bars are sites of violent ongoing star formation. On the other hand, bars induce large-scale gas motions with shocks which are observed due to dust lanes. Can these two features - violent star formation and supersonic gas dynamics with shocks - be related to each other in barred galaxies?

\section{Geometry of the Bar-Induced Shocks}

Analytical studies and especially computer simulations of the gas dynamics induced by bars (see, for instance, the papers by Athanassoula and by Teuben in these proceedings) indicate that supersonic gas motions with shocks occur as the response of the interstellar gas to a bar gravitational potential. The shock front usually forms two-armed spiral structure. The inner parts of the shock front surface curve around the center of the disk. This surface is concave to the up-flow.

Producing the shocks, the bar acts as a "gravitational piston". But when the gas comes into motion, it behaves more and more independently of gravitation, and the nonlinear laws of gas dynamics control them mostly. While there are no "standard" solutions for shocks of such a geometry, some indications concerning their structure and evolution can be driven from general gas dynamics concepts using two gas dynamical patterns: stability of the plane shocks and shrinking of cylindrical shocks.

The plane front is known to be very stable against any perturbations, and in particular against deformations of the geometry of its surface. A concave segment of a bar-induced shock may be considered as a perturbation. The front tends to get flattened, if the radius of curvature of the segment surface is much greater than the size of the segment. One can expect that the tendency for 
flattening can reveal itself in the evolution of the outer slightly curved parts of the bar-induced shocks.

\section{Shrinking and Instability}

The inner highly curved parts of the bar-induced shocks should evolve in a quite different way. The radius of curvature is comparable with the size of the inner segment of the shock. The pattern of the curved cylindrical shocks indicates that the shocks of this type should be shrinking and their motion to the center of curvature should be accelerating. According to the Guderley (1942) solution for the shrinking cylindrical shocks, the shock velocity tends to infinity when the distance from the axis of symmetry (or from the center of curvature of each segment of the front) tends to zero. But the shrinking process is limited by hydrodynamical instabilities which destroys the Guderley regime well before the center of curvature is reached. The physical cause for the instability is due to the acceleration of the shock (Gurevich \& Rumjantsev 1970, 1973, Chevalier 1976). The shrinking and accelerating shocks decay which leads to formation of turbulence zones behind their fronts.

These results - with all the necessary reservations - may be applied to the central parts of the spiral shocks produced by the bars. While the shock surface is not closed in this case, the evolution of the non-stationary highly curved shock segment should lead to its shrinking, acceleration, and subsequent hydrodynamical instability.

\section{Star Formation}

Basing on these considerations, one can imagine that soon after their formation by the gravitational piston of the bar, the inner concave part of each of the shock arms will produce compression of the gas, and the shocked gas will finally be turbulent due to hydrodynamical instability. The high density and high velocity dispersion of the gas provide suitable physical conditions for star formation. In this way, two large-scale regions of star formation may form near the edges of the bar.

The star formation and the evolution of stars in these two regions develop in a similar way and more or less synchronously. These processes can give rise to a new chain of violent gas dynamic processes in the bar area.

In each of the regions, strong stellar winds and multiple supernova explosions can produce a shell with a face shock. This shell is expanding. Two shells of such an origin at two edges of the bar may come into contact and collide with each other. The physics of this process is studied by Chernin et al. (1995).

Two reflected shocks form as a result of the collision. They propagate back to the initial regions of star formation and drive fresh shocked gas to these regions. And again high gas density (and high velocity dispersion in the gas) can provide conditions for new bursts of massive star formation in the same regions. One can expect that such regions may reveal in observations two populations of young stars, the Cepheids and OB stars, with the gap in the ages of the populations of about $30-50$ Myrs. 
Acknowledgments. I am grateful to Yury Efremov for discussions of the problem. The support by the IAU and LOC for attending the conference is appreciated.

\section{References}

Chernin, A. D., Efremov, Y. N., \& Voinovich P. A. 1995, MNRAS, in press Chevalier, R. A. 1976, ApJ, 207, 872

Guderley, G. 1942, Luftfahrtforschung, 19, 32

Gurevich, L. E. \& Rumjantsev, A. A. 1970, JETP, 58, 1395

Gurevich, L. E. \& Rumjantsev, A. A. 1973, Ap\&SS, 12, 115 